\title{
When pharmacotherapeutic recommendations may lead to the reverse effect on physician decision-making
}

\author{
Abilio C. de Almeida Neto · Timothy F. Chen
}

Received: 28 February 2007/ Accepted: 1 June 2007/Published online: 22 June 2007

(C) Springer Science+Business Media B.V. 2007

\begin{abstract}
For long the medical literature has shown that patients do not always receive appropriate care, including pharmacotherapeutic treatment. To achieve improved patient care, a number of physician-oriented interventions are being delivered internationally in an attempt to implement evidence based medicine in routine daily practice of medical practitioners. The pharmacy profession has taken an active role in the delivery of intervention strategies aimed at promoting evidence based prescribing and improved quality and safety of medicine use. However, the medical literature also supports the notion that valid clinical care recommendations do not always have the desired impact on physician behaviour. We argue that the well-established theory of psychological reactance might at least partially explain instances when physicians do not act upon such recommendations. Reactance theory suggests that when recommended to take a certain action, a motivational state compels us to react in a way that affirms our freedom to choose. Often we choose to do the opposite of what the recommendation is proposing that we do or we just become entrenched in our initial position. The basic concepts of psychological reactance are universal and likely to be applicable to the provision of recommendations to physicians. Making recommendations regarding clinical care, including pharmacotherapy, may carry with it implied threats, as it can be perceived as an attempt to restrict one's freedom of choice potentially generating reactance and efforts to avoid them. By identifying and taking into
\end{abstract}

A. C. de Almeida Neto $(\bowtie)$. T. F. Chen

Faculty of Pharmacy, The University of Sydney, Sydney, NSW 2006, Australia

e-mail: abilio@pharm.usyd.edu.au account factors likely to promote reactance, physicianoriented interventions could become more effective.

Keywords Pharmacist - Physician - Psychological reactance $\cdot$ Medication review $\cdot$ Professional behavior change $\cdot$ Interventions $\cdot$ Continuing medical education . Health psychology $\cdot$ Decision support systems

It is well known that the use of medicines often results in avoidable complications or adverse events. In the United States a study showed that approximately $60 \%$ of iatrogenically related admissions to intensive care units were drug related and $75 \%$ were preventable [1]. In Australia, adverse medicine events are responsible for $1.6 \%$ of all hospital admissions [2].

To achieve improved quality and safety of medicine use, a number of physician-oriented interventions are being delivered internationally in an attempt to improve evidence based prescribing in routine daily practice of medical practitioners-e.g., distribution of educational materials, decision support systems, outreach visits, medication reviews, etc.

The pharmacy profession has taken an active role in the delivery of intervention strategies related to pharmacotherapy. For example, in medication review programmes, pharmacists interview patients, conduct assessments of information gathered on patients' use of medicines and prepare reports for physicians, which may contain pharmacotherapeutic recommendations [3]. Pharmacists also are typically involved in educational outreach programmes. In such programmes it is common for pharmacists to take up the role of educator, visiting physicians in their office to provide educational interventions on specific pharmacotherapeutic topics. As pharmacists and physicians share 
expertise in pharmacotherapy, their involvement in intervention strategies aimed at promoting quality and safety of medicine use makes sense. Indeed, the literature has reported positive results on patient health outcomes arising from such interventions $[4,5]$.

However, despite the delivery of interventions designed to foster quality and safety of medicine use, the literature provides support for the notion that, at times, despite physicians agreeing to the value of evidence based clinical recommendations, intervention strategies do not always have an impact on physician behaviour. For example, in a study in which both physicians and community pharmacists had negotiated actions based on medication reviews, the researchers were only able to document a rate of implementation of $42 \%$ of the proposed actions [6]. These findings are supported by our own experience working with community pharmacists involved in medication management programmes in Australia. Some of these pharmacists report that sometimes their recommendations have the desired impact on physician choice of pharmacotherapy, but at times they have no impact at all and in some instances they even cause physicians to respond negatively.

As expected, failure to change physician behaviour is not restricted to the prescribing area. Indeed, non-compliance to evidence based recommendation is a consistent finding in clinical care research [7]. For example, Grol and Grimshaw [7] note that hospital-acquired infections affect one in every 11 patients with $13 \%$ mortality rate and that $15-30 \%$ of infections are considered to be preventable by handwashing [8]. Still, compliance with handwashing by health care providers, in particular medical practitioners, is known to be poor [7]. Indeed, with surprising frequency, and to the considerable dismay of interventionists, physician-oriented interventions often fail to improve handwashing behaviour [7, 9]. Without a doubt while there are certainly circumstances that may lead physicians to justifiably question clinical care recommendations, in general non-compliance to evidence based recommendations proves costly to patients and society alike.

Currently, there is a lack of theoretical framework for understanding instances when valid recommendations do not lead to the desired impact on physician behaviour. The key question is: What are the barriers that impede physician acceptance of valid recommendations? We argue that the theory of psychological reactance [10] might at least partially explain instances when evidence based recommendations fail to impact on physician behaviour.

\section{Psychological reactance}

Psychological reactance is a tendency to resist perceived attempts by others to control the individual's behaviour
[10]. Most of us like to perceive ourselves as being in control; we value our sense of freedom and autonomy and like to project an image of being in control [11]. In general, when we perceive that our freedom of choice is being restricted by the action of others, we react in a way that affirms our ability to choose. Often we choose to do the opposite of what the recommendation is proposing that we do or we just become entrenched in our initial position. This phenomenon is called psychological reactance [10]. Reactance is directed towards reattaining the restricted freedom; a motivational state compels us to reassert that freedom [10]. In many circumstances, reactance results in an increase in the attractiveness of the constrained behaviour and an increased sense of confidence in the ultimate decision made [10]. Particular noteworthy is the finding that reactance appears to be a biological tendency, an inherent human characteristic. At the very least, it can be said that reactance manifests itself at a very young age [12].

For the last four decades the theory of psychological reactance has been widely tested and applied with reactance being widely recognised as a powerful determinant of behaviour. To date, studies across a vast array of behaviours have consistently demonstrated that recommending that individuals take a certain course of action often produces the opposite effect, consistent with this tendency to resist perceived attempts by others to restrict freedom of choice. For example: in the marketing area research indicates that expert recommendations are desirable as long as they are consistent with the choice tendency of individuals. When expert recommendations contradict the consumer's choice tendencies, a reactant state is activated on the part of the decision maker leading to a behavioral "backlash" that results not only in consumers ignoring the recommendations but in intentionally contradicting them [13]. Not only there is an increase in choice of the non-recommended option, but also a significant increase in confidence in the non-recommended option [13].

In the healthcare literature, psychological reactance has been primarily used to explain patient non-compliance, i.e., why some patients refuse to cooperate with medical treatment [14, 15]. Fogarty and Young [15] conducted research that indicates that individual variability in compliance to a medication regimen is related to individual variability in reactance as a personality trait [15]. Psychological reactance has also for long been observed in the natural setting in response to perceived attempts to restrict one's freedom of choice. A classic example of psychological reactance in the everyday life took place when a city in the United States banned the possession and use of laundry detergents containing phosphates for environmental reasons. It was found that residents stockpiled an enormous quantity of detergents containing phosphates before the ban went into effect. Others organised carpools to neighbouring counties 
to purchase detergents containing phosphates $[16,17]$. As a result of the ban, the perceived value of the prohibited products was increased; compared with residents in neighbouring counties, who were free to choose whatever detergent they liked, residents subjected to the ban rated phosphate detergents as gentler, easier to pour, better whiteners and stain removers, and more effective in cold water. It is noted that phosphates have no impact whatsoever on the cleaning effectiveness of detergents.

Psychological reactance and physician-oriented interventions

The long history of successful support for reactance theory in diverse settings and areas of behaviour may also have implications for physician-oriented interventions in which evidence based clinical recommendations are provided, as health care providers may not be immune to the phenomenon of psychological reactance. It is true that the basic concepts of psychological reactance are universal and likely also to be applicable to the provision of evidence based clinical recommendations to physicians.

In the context of physician-oriented interventions, the theory and research on psychological reactance would predict that at times when opposing recommendations are provided, physicians' perceptions of threats to their freedom to select an appropriate course of action could produce the opposite reaction leading them to become more likely to ignore the recommendation or to choose the non-recommended option. This would be the case even in circumstances where physicians may agree, in essence, to the value of the recommendation.

...to the extent that it is perceived by the individual that the communicator is trying to make him change, his freedom to decide for himself will be threatened and he will experience reactance... Information and arguments can be quite helpful to the individual and may result in positive influence, but the perception that the communicator is attempting to influence will tend to be seen as a threat to one's freedom to decide for oneself ([10], p. 94 cited in [14]).

Indeed, making recommendations regarding clinical care, including pharmacotherapy, may carry with it implied threats, as it can be perceived as an attempt to restrict one's freedom of choice generating reactance and efforts to avoid them. Furthermore, the inherent "veto" power that physicians have over the treatment regimen may facilitate manifestation of reactant-style responses making noncompliance to evidence based recommendations more likely to occur.

It is also important to recognise that even in circumstances where physicians agree to participate in interventions, they may not necessarily expect their practice to be challenged. For example, there are several possible reasons for a physicians agreeing to a medication review. In some cases, physicians may wish to monitor the individual patient's self-medication habits or they may be concerned about possible patient non-compliance with the medication regimen.

It is also true that physicians have for long subscribed to a body of ethical rules created above all for the benefit of the patient [18]. Professional behaviour of medical practitioners is guided by these ethical rules, which dictate what is morally approved and disapproved conduct specifying what ought to be done [18]. It is reasonable to expect medical ethics to have a considerable impact on physician decision-making in situations that involve potential harm to the patient. For example, the use of a non-steroidal anti-inflammatory drug (NSAID) in a patient taking the anticoagulant warfarin puts the patient at an increased risk of bleeding. In such situations, which involve potential harm to the patient, recommendations arising from intervention strategies most certainly will be recognised by the physician as an injunctive professional norm (a norm of "what ought to be done") and most certainly will be implemented. However, it is possible that providing physicians with valid opposing clinical care recommendations that do not involve potential harm to the patient may at times be counterproductive. For example, in a patient with low gastrointestinal and cardiovascular risk, recommendation to use a non-selective NSAID if the patient is responding well to a COX-2 selective NSAID, may be perceived as an unwarranted attempt to restrict one's action, increasing the likelihood of a reactant-style response.

Although no study appears to have specifically addressed reactance from a health care providers' perspective, a recent qualitative study has provided some indication that reactance might play a role in physician decision-making in response to pharmacotherapeutic recommendations [19]. This study investigated determinants of successful collaboration between community pharmacists and physicians in medication review. Participating pharmacists discussed the need to be diplomatic in their recommendations, as they believed physicians' misperception that they were "telling them what to do" could induce dismissal of their recommendations.

\section{Possible determinants of reactance}

It is possible that reactant responses may be more likely to occur in situations where the physician has not requested the information/recommendation. Experiments outside the medicines area have demonstrated that unsolicited information can evoke psychological reactance even when 
recommendations provide a substantial amount of information beyond what the decision maker knows [20]. Interestingly enough, in situations where the recommended action went contrary to the individual's choice tendency, this reactance to unsolicited information was significantly more likely to occur when recommendations came from a source perceived to be an expert [20]. The authors suggest that unsolicited recommendations may be perceived as more of a threat or an intrusion when they come from credible sources. It is noted that although unsolicited information is typically provided to physicians through written materials and computerised decision support systems, in some programmes medicines use review can be initiated by the pharmacist and report sent to the patient's physician in an unsolicited fashion [21].

It is also possible that the perception that the particular interventionist is directing recommendations specifically to the particular physician, as opposed to the treatment of the patient's condition in general, also could make reactance more likely to occur. Brehm [10] demonstrated that reactant responses are more likely to occur when individuals perceive the constraint as being specifically directed at them as opposed to when it is perceived to be impersonal [10]. If indeed, this is found also to apply to physicianoriented interventions, it may have implications for how pharmacists and other health care professionals provide recommendations to physicians.

Sense of medical professionalism might also be implicated in reactant responses. We do value our sense of freedom and autonomy and we like to project an image of being in control [11]. Indeed, physicians are trained to make sound decisions with regard to treatment regimen and it is reasonable to expect them to believe that they are in control and have the knowledge and skills required to make the best possible decisions. The acceptance of a contrary recommendation to what is being used to treat the patient's illness could undermine the physician's own sense of competence, reducing the cognitive basis of professionalism. On the other hand, dismissal of the recommended option could have the opposite effect reinforcing physician's ability to choose the most appropriate treatment.

We also know that once aroused, reactance may heighten individual's sensitisation to additional threats to freedom of control [22]. It is also reasonable to believe that at a time when governments are encouraging greater involvement of allied healthcare professionals in patient care, issues related to professional territoriality may sensitise physicians to reactance in response to recommendations from allied healthcare professionals, including the pharmacist. Although the literature reports positive medical profession's attitudes to pharmacists and their clinical role, some sentiments of encroachment are supported by studies that found that not all physicians view favourably greater involvement of pharmacists in patient care [23-25]. Thus, the physician-pharmacist interaction in the intervention process could be burdened with social and personal pressures, which can increase the possibility of a reactant response. Indeed, psychological reactance may offer a theoretical framework that could enhance our understanding of the, at times, difficult relationship between pharmacists and physicians.

It is also possible that the nature of the physician relationship with the allied healthcare professional is also a determinant of reactance. For example, it has been argued that the most successful pharmacist interventions have been those in which the pharmacist works in close liaison with the physician [26]. Indeed, in the United States much of the evidence supporting pharmacist involvement in collaborative drug therapy management is derived from the ambulatory care setting [27] where routine contact with physicians may allow for the development of the trusting relationship needed for interprofessional collaboration [28].

Finally, another factor that may determine reactant responses to recommendations include individual differences, as some people are more likely to have a reactant response in general to any perceived constraint [29]. Individual variations in susceptibility to reactance can explain why identical situations may lead to markedly different perceptions of threat and magnitudes of reactant responses in different persons. Indeed, scales developed to measure reactance as a trait demonstrate that individual reactance levels lie along a continuum from very low to very high [29]. Naturally, in the context of physician-oriented interventions, the nature and intensity of the reactant response is also likely to be a function of how much the particular physician values his/her freedom to select the treatment option him/herself. Again, a recent qualitative study provides some support to the notion that some physicians are more prone to psychological reactance than others [19]. In this study, personality of medical practitioners was discussed as a factor influencing the outcome of medication review. Participating pharmacists distinguished between physicians who they thought were amenable to discussing their prescribing decisions and those who were not. These results indicate that interventionists do assess the reactance potential of the individual physician although it is unlikely that they label the assessment as such. It is noteworthy that when interventionists sense reactance in their interaction with the physician, they may be faced with a difficult dilemma, as they are aware of the possibility that the provision of evidence based clinical recommendation may trigger the opposite response. What can the interventionist do in such situation? 
Dealing with reactance

A phenomenon like psychological reactance, which is likely to impede the desired outcome of physician-oriented interventions, needs to be adequately addressed when training healthcare professionals to deliver such interventions. We must be mindful that the process of delivering physician-oriented interventions can be difficult as psychological reactance is not a reasonable or rational response; it can cause people to become highly emotional and motivated to defend their behaviour.

Prior research on reactance suggests that individuals' ability to exercise some choice (freedom) in relation to recommendations may impact on compliance to the advice provided. That is, maximising the individual's perception of free choice can minimise reactance [22]. It is possible that the degree to which the physician is incorporated in the selection of evidence based clinical care practices may affect the extent to which the intervention will induce reactance. Indeed, it is possible that intervention strategies which give physicians the impression they have little or no say on their own clinical practices will be less likely to be effective, as they are perceived as more threatening, compared to those where physicians perceive themselves as exercising some choice.

We are now training pharmacists on how to deal with psychological reaction in the context of medication review. We also expect such training to enhance commitment to the pharmacy lead intervention from the interventionist's perspective, as after being equipped with tools for dealing with reactance, pharmacists may experience a greater sense of self-efficacy in dealing with all types of physicians, including those who are highly reactant. In addition, awareness of reactant responses and its causes may assist pharmacists and other healthcare professionals in altering their emotional response to reactant behaviour avoiding a decreased sense of self-efficacy.

\section{Concluding remarks}

The current paper addressed an intrinsic human characteristic, psychological reactance, as a factor that may influence the outcome of physician-oriented intervention strategies, including those typically delivered by pharmacists. Although psychological reactance has not been tested from a healthcare provider's perspective, there is enough research to suggest the basic cognitive process represented is universal and also valid in the context of clinical care recommendations in the healthcare setting. Indeed, reactance theory fits well as it offers some explanation of why at times valid recommendations fail to have the desired impact on physician behaviour.
It is possible that as human beings, even healthcare professionals are subjected to the same human emotions and weaknesses as humans in general. However, a role for reactance in clinical care recommendations needs to be investigated empirically. If this role is established, factors likely to increase the potential for reactance may need to be taken into account when implementing intervention strategies that attempt to influence physician behaviour.

It also is acknowledged that physician non-compliance to valid recommendations is more likely to be a complex, multi-faceted phenomenon. Physicians may not adhere to evidence based recommendations for a number of reasons unique to the individual patient, and the circumstance. It is most likely that reactance, although a powerful determinant of behaviour in general, is only one of the many factors that influence decision-making in relation to clinical care/ pharmacotherapeutic recommendations.

\section{References}

1. Darchy B, Le Miere E, Figuérédo B, Bavoux E, Domart Y. Iatrogenic diseases as a reason for admission to the intensive care unit. Arch Intern Med 1999;159:71-8.

2. Australian Government. Department of Health and Ageing. Australian Phamaceutical Advisory Council. 2005. Guiding principles to achieve continuity in medication management. http://www.health.gov.au/internet/wcms/publishing.nsf/Content/ nmp-guiding (30 January, 2007).

3. Australian Government. Department of Health and Ageing. 2005. Domiciliary medication management-Home medicines review: helping your patients manage their medicines at home. http:// www.health.gov.au/internet/wcms/publishing.nsf/Content/healthepc-dmmr.htm/\$FILE/dmmrguidelines.pdf (31 January, 2007).

4. Sorensen L, Stokes JA, Purdie DM, Woodward M, Elliott R, Roberts MS. Medication reviews in the community: results of a randomized, controlled effectiveness trial. Br J Clin Pharmacol 2004;58(6):648-64.

5. Zermansky AG, Alldred DP, Petty DR, Raynor DK, Freemantle $\mathrm{N}$, Eastaugh J, Bowie P. Clinical medication review by a pharmacist of elderly people living in care homes-randomised controlled trial. Age and Ageing 2006;35(6):586-91.

6. Gilbert A, Roughead EF, Beilby J, Mott K, Barrat JD. Collaborative medication management services: Improving patient care. Med J Aust 2002;177:189-92.

7. Grol R, Grimshaw J. From best evidence to best practice: effective implementation of change in patients' care. Lancet 2003;362(9391):1225-30.

8. Stone S. Hand hygiene: the case for evidence base education. J R Soc Med 2001;94:278-81.

9. Dorsey ST, Cydulka RK, Emerman CL. Is handwashing teachable?: failure to improve handwashing behavior in an urban emergency department. Acad Emerg Med 1996;3:360-5.

10. Brehm JW. A theory of psychological reactance. New York, NY: Academic Press; 1966; ISBN-10: 0121298507.

11. Barr R, Hinkle S, Smith K, Fenton M. Reactance as a function of actual versus projectedutility. J Pers Soc Psychol 1980;38:41622.

12. Brehm J, Weintraub M. Physical barriers and psychological reactance: 2-year-olds response to threats to freedom. J Pers Soc Psychol 1977;35:830-836. 
13. Fitzsimons GJ, Lehmann DR. Reactance to Recommendations: When Unsolicited Advice Yields Contrary Responses. Mark Sci 2004;23(1):82-94.

14. Fogarty J. Reactance Theory and Patient Noncompliance. Soc Sci Med 1997;45(8):1277-88.

15. Fogarty J, Youngs Jr GA. Psychological reactance as a factor in patient noncompliance with medication taking: a field experiment. J Appl Soc Psychol 2000;30(11):2365-91.

16. Mazis MB, Settle RB, Leslie DC. Elimination of phosphate detergents and psychological reactance. J Mark Res 1973;10:390-5.

17. Mazis MB. Antipollution measures and psychological reactance theory: a field experiment. J Pers Soc Psychol 1975;(31):654-66.

18. American Medical Association. 2005. Principles of medical ethics. http://www.ama-assn.org/ama/pub/category/2512.html (25 April 2006).

19. Ghaly J, Chen T, de Almeida Neto A. Interprofessional collaboration in home medicines review: pharmacists' perspective. Faculty of Pharmacy. Sydney, The University of Sydney. Honours Thesis in Pharmacy; 2006.

20. Fitzsimons GJ, Lehmann DR. 2001. Unsolicited Advice as an Implicit Choice Restriction: When Intelligent Agent Recommendations Yield Contrary Responses. http://www-marketing.wharton.upenn.edu/ideas/pdf/01-008.pdf (25 April 2006).

21. Bellingham C. How to offer a medicines use review. Pharm J 2004;273:602.
22. Brehm SS, Brehm JW. Psychological reactance: A theory of freedom and control. New York, NY: Academic Press, 1981; ISBN 012129840X.

23. Spencer J A, Edwards C. Pharmacy beyond the dispensary: general practitioners' views. BMJ 1992;304(6843):1670-2.

24. Ranelli PL, Biss J. Physicians' perceptions of communication with and responsibilities of pharmacists. J Am Pharm Assoc 2000;40(5):625-30.

25. Tanskanen P, Airaksinen M, Tanskanen A, Enlund H. Counselling patients on psychotropic medication: physicians' opinions on the role of community pharmacists. Pharm World Sci 2000;22(2):59-61.

26. Holland R, Smith R, Harvey I. Where now for pharmacist led medication review? J Epidemiol Comm Health 2006;60:92-3.

27. Hammond RW, Schwartz AH, Campbell MJ, Remington TL, Chuck S, Blair MM, Vassey AM, Rospond RM, Herner SJ, Webb CE. American college of clinical pharmacy position statement: collaborative drug therapy management by pharmacists-2003. Pharmacother 2003;23(9):1210-25.

28. Chen TF, de Almeida Neto AC. Exploring elements of interprofessional collaboration between pharmacists and physicians in medication review. Pharm World Sci (In Press).

29. Hong SM. Hong's Psychological Reactance Scale: a further factor analytic refinement. Psychol Rep 1992;70:512-4. 